\title{
Relationship Between Mesostructure, Mechanical Behaviour and Damage of Cement Composites Under High-Pressure Confinement
}

\author{
P. Forquin • A. Arias • R. Zaera
}

\begin{abstract}
This work seeks to determine how the mesostructure of seven types of cement composites containing alumina particles or not controls their deviatoric strength, compaction law, and damage under high-pressure of confinement. First, the method of analysis of quasioedometric compression tests is presented. Accuracy of the method for concrete is discussed by means of numerical simulations. The confined compression tests performed show the effects of adding ceramic aggregates. Furthermore, an application of post-mortem analysis by infiltration technique of each specimen is presented, revealing a highly micro-cracked pattern depending of the mesostructure of these materials. From these observations, a discussion is presented on the influence that the addition of ceramic aggregates exerts on the confined behaviour of these composites. The tests showed a highly beneficial effect of the presence of particles, on both the deviatoric strength and the compaction law of the concretes considered.
\end{abstract}

Keywords Mortar and concrete · Ceramics · Cracking · Confined compression $\cdot$ Pressure sensitivity

\footnotetext{
P. Forquin $(\bowtie)$

Laboratory of Physics and Mechanics of Materials,

UMR CNRS 75 54, University Paul Verlaine of Metz,

Ile du Saulcy,

57045 Metz cedex, France

e mail: forquin@univ metz.fr

A. Arias $\cdot$ R. Zaera

Department of Continuum Mechanics and Structural Analysis,

University Carlos III of Madrid,

Avda. de la Universidad 30,

28911 Leganés, Madrid, Spain
}

\section{Introduction}

In the 1980s, the appearance of high- or very-highperformance concretes with average failure stress under simple compression of $60-120 \mathrm{MPa}$ [1], and then the emergence of ultra-high-performance concretes of over $200 \mathrm{MPa}$ in the 1990s [2], have stimulated studies concerning the mechanical behaviour of these materials and their applications [3-5]. In particular, because of their easy use and high hardness, these types of materials are attractive as structural and impact-resistant materials [6, 7]. The necessity to protect army bunkers, nuclear power plants, and airport runways from missile impact, or civil buildings from natural hazards such as tornadoes or earthquakes has prompted several studies on concrete under extreme conditions [8-10].

In a concrete structure subjected to shock or impact, severe hydrostatic compression is found near the impact location and a field of confined compression is created ahead of the projectile, which produces irreversible reduction of the volume $[5,11,12]$. The resistance of the material under high pressure, the law of compaction (evolution of volumetric strain) and the elastic parameters, condition the penetration of the projectile into the target $[10,13,14]$. The analysis of the concrete subjected to this type of impact loading implies knowing the effect that high-pressure confinement exerts on the mechanical properties [5, 10-12].

Extensive literature describes mechanical properties of brittle materials under pressure, ever since the pioneer work of Bridgman [15]. Thus, the role of the hydrostatic pressure on the failure of brittle materials has been investigated by Paterson [16], Heard and Cline [17], Horii and NematNasser [18], and more recently by Chen and Ravichandran [19], Ramsey and Chester [20] and Clifton [6]. These works pointed out that under high confinement stresses, 
brittle materials such as ceramics can deform plastically. This transition to ductile behaviour has also been reported by Rittel and Brill [21] for a brittle polymeric material (PMMA). Thus, confinement of brittle materials such as concrete may cause their failure by ductile mechanisms after large inelastic deformations. Since these high pressures typically arise in front of a penetrator making impact against armour, the knowledge of the behaviour of materials at high pressures is of great concern for armour applications. Therefore, to understand the high-pressure response of brittle materials such as concrete involves considering aspects of the behaviour of ductile materials $[6,21]$.

The strength of concretes under confined compression can be characterized by triaxial compression tests or by quasi-oedometric compression tests. In the triaxial compression tests, hydrostatic pressure is applied to a cylindrical specimen by a fluid under pressure and the cylinder is then subjected to axial compression [22, 23]. The deviatoric stress is then measured as a function of the axial strain under different confining pressures, and the tests have shown the ductility of concretes under strong confinement and the rise of strength with pressure. However, it is noticeable that when the stress difference reaches its elastic threshold, it is kept more or less constant while the axial strain increases up to $6 \%$ to $10 \%$ [24-26]. Therefore, the strength of concretes appears much more pressure-dependant than sensitive to the axial strain and this remark is a basic hypothesis which is required to deduce the confined behaviour of concrete materials from a single quasioedometric compression test [12]. The triaxial compression tests are not without their limitations and difficulties, however; they require a very-high-pressure chamber of 1,000-10,000 bars [27] and water-tightness between the fluid and the specimen that can be difficult to achieve.

Another method, applied to test metals [28, 29], ceramics $[19,30]$, polymers [21, 31] and concretes [12, 32-34], consists in confining a cylindrical specimen enclosed in a metal vessel (Fig. 1). In the course of the axial compression of the sample, the specimen tends to expand under the effect of the radial swelling and to exert pressure on the confining vessel. The test is called quasi-oedometric because the radial expansion is not absolutely nil but it is considerably limited by the stiffness of the vessel. Both the axial and the radial stress in the sample are raised during the test. The lateral confinement pressure is registered by means of gauges attached to the outer surface of the vessel (Fig. 1), so the evolution of the strength of the material can be deduced over a wide range of confinement pressures. The difficulties of triaxial compression tests do not appear during a quasioedometric compression test since the lateral pressure is applied directly by the vessel.

The present paper focuses on the study of quasioedometric compression test on seven concretes performed under quasi-static conditions. Quasi-static conditions have been applied in other works to study the effect of confinement on metal and polymers [28, 29] and specifically on cement materials $[10,12,33]$. Moreover, data and parameters identified in similar quasi-static confined compression experiments have been used in numerical predictions of the impact behaviour of concrete [5, 35]. Some authors have reported the beneficial effect of incorporating high-strength particles to improve the impact resistance of concrete materials or brittle polymers, particularly when particle size is very close to the projectile calibre $[8,36$, 37]. In this work, the concretes have been developed containing alumina particles or not [4], in order to analyse their influence on the mechanical behaviour under high pressure. The experimental data showed an improvement of spherical and deviatoric behaviour of the considered cement composites.

Additionally, an application of post-mortem analysis by infiltration technique of each specimen is presented. This methodology, applied commonly to the analysis of damage in brittle materials, can highlight worthwhile points concerning the influence of a mesostructure on the behaviour under confined compression. This procedure can be extended to other kinds of aggregates mixed with other types of matrices (metal or polymer).

\section{Method of Analysis of the Quasi-oedometric Compression Test}

The principles of the test and of its analysis have been explained by Forquin et al. [12]. Three hoop-strain gauges
Fig. 1 Sketch of the quasioedometric compression test set-up

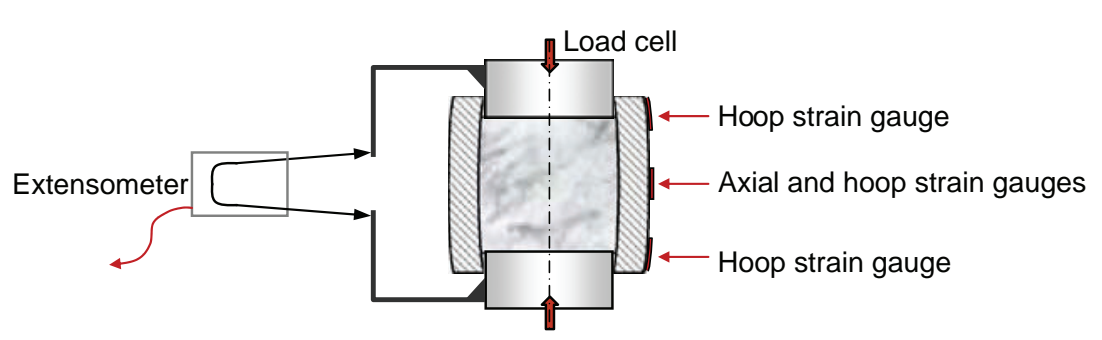


attached to the confinement vessel enable a deduction to be made concerning the radial strain and the radial stress in the specimen tested. The relation between the radial stress within the specimen, and the hoop strain of the external wall, is deduced from calculations made with Abaqus/ Standard [38]. In these calculations, a pressure is applied along a height $h_{\text {press }}\left(h_{\text {press }}=34\right.$ or $\left.40 \mathrm{~mm}\right)$ on the internal wall of the vessel. The behaviour law used to model the steel of the vessel is determined by tensile tests on samples which were cut from the original metal bar and tested by a $100 \mathrm{kN}$ Instron ${ }^{\circledR}$ machine [12]. From the calculations, the evolution of the radial stress is determined as a function of the external-hoop strain at the symmetry plane $(z=0)[12]$ :

$\bar{\sigma}_{\text {radial }}\left(h_{\text {press }}=40\right)=f_{40}\left(\varepsilon_{\theta \theta}^{(z=0, \text { ext })}\right)$

$\bar{\sigma}_{\text {radial }}\left(h_{\text {press }}=34\right)=f_{34}\left(\varepsilon_{\theta \theta}^{(z=0, \text { ext })}\right)$

From these two equations, we deduce the internal radial stress as a function of the axial strain, using the following linear ratio:

$$
\begin{aligned}
\bar{\sigma}_{\text {radial }}\left(\bar{\varepsilon}_{\text {axial }}, \varepsilon_{\theta \theta}^{(z=0, \text { ext })}\right)= & \left(1-\frac{\bar{\varepsilon}_{\text {axial }}}{\varepsilon_{\text {ref }}}\right) f_{40}\left(\varepsilon_{\theta \theta}^{(z=0, \text { ext })}\right) \\
& +\left(\frac{\bar{\varepsilon}_{\text {axial }}}{\varepsilon_{\text {ref }}}\right) f_{34}\left(\varepsilon_{\theta \theta}^{(z=0, \text { ext })}\right)
\end{aligned}
$$

in which $\bar{\varepsilon}_{\text {axial }}$ is the nominal axial strain, $\varepsilon_{\text {ref }}$ the reference strain, and $f_{40}\left(\varepsilon_{\theta \theta}^{z=0, \text { ext }}\right)$ and $f_{34}\left(\varepsilon_{\theta \theta}^{z=0 \text {,ext }}\right)$ are the functions of equations (1) and (2). Thus, the lateral pressure introduced in the numerical simulation for a different value of $h_{\text {press }}$ was compared to the radial stress given by equation (3). The difference was less than 5\% when the axial strain did not exceed $20 \%$ (in absolute terms). This condition was consistently fulfilled for the tests performed in this study and, in accordance with a previous work [12], the reference strain $\left(\varepsilon_{\text {ref }}=-0.15\right)$ was chosen to limit the interpolation error.

In the same way, these calculations provide a relation between the external and the internal hoop strains of the vessel [12]. An expression was deduced for the average internal radial strain of the specimen in the vessel as a function of the axial strain and two strains $\left(\varepsilon_{\theta \theta}^{(z=0, \text { ext })}, \varepsilon_{\theta \theta}^{(z=18, \text { ext })}\right)$ measured in the tests by strain gauges attached to the vessel:

$\bar{\varepsilon}_{\text {radial }}\left(\bar{\varepsilon}_{\text {axial }}, \varepsilon_{\theta \theta}^{(z=0, \text { ext })}, \varepsilon_{\theta \theta}^{(z=18, \text { ext })}\right)$

In addition, the load cell of the hydraulic press gives access to the average axial stress $\left(\bar{\sigma}_{\text {axial }}\right)$ imposed on the specimen. Since the radial strain and the internal radial stress in the concrete are known, the deviatoric stress $\left(\bar{\sigma}_{\text {deviatoric }}\right)$ and the hydrostatic pressure can be deduced as:

$\bar{\sigma}_{\text {deviatoric }}=\left|\bar{\sigma}_{\text {axial }}-\bar{\sigma}_{\text {radial }}\left(\bar{\varepsilon}_{\text {axial }}, \varepsilon_{\theta \theta}^{\text {ext }}\right)\right|$

$\bar{p}_{\text {hydrostatic }}=-\frac{1}{3}\left(\bar{\sigma}_{\text {axial }}+2 \bar{\sigma}_{\text {radial }}\left(\bar{\varepsilon}_{\text {axial }}, \varepsilon_{\theta \theta}^{\text {ext }}\right)\right)$

and the volumetric strain is given by the formulae:

$\bar{\varepsilon}_{\text {volumetric }}=\left(1+\bar{\varepsilon}_{\text {axial }}\right)\left(1+\bar{\varepsilon}_{\text {radial }}\right)^{2}-1$

Thus, with only the data of the axial force, the axial strain, and the external hoop strain measured by the three gauges attached to the vessel, we can determine the evolution of the deviatoric stress (deviatoric behavior) and the variation of the volumetric strain with the hydrostatic pressure (volumetric behavior).

\section{Quasi-oedometric Compression Tests of Seven Cement Composites}

Elementary Mechanical Properties of the Cement Composites

Seven materials were prepared and tested under quasioedometric compression. Two cement- based matrices named M1 and M2 (see the terminology and composition in Table 1) were used. In the M2 mortar, the silica fume was added, representing 10\% in weight of the amount of cement. These matrices give a satisfactory relation between production cost, malleability and strength even when particles are added. Particles were spherical (diameter $4 \mathrm{~mm}$ ) or angular (size of 3 to $6 \mathrm{~mm}$ ). The cylindrical and cubic samples used in the study were cut and extracted from the interior of two large blocks around $280 \times 200 \times 60 \mathrm{~mm}^{3}$, one from each material. Figure 2 shows the surface of each concrete specimen at the same scale. The density of the matrix of each material $\left(\rho_{\mathrm{M}}\right)$ has been estimated in a previous work [4] from their density $\left(\rho_{\mathrm{C}}\right)$ and from the mass fraction of the particles used $\left(f_{\mathrm{Mp}}\right.$; Table 1$)$. The strength values found under bending and simple compression tests have also been determined [4] and are reported in Table 1.

The results of three-point bending tests performed show that the failure of cement composites made of matrix M1 (M1M and M1Sph) was probably due to particle/matrix decohesion leading to a lower average strength. On the contrary, particles did not weaken the bending strength of the particulate M2 mortar. The M2S, M2M and M2Sph average strengths were even higher than that of the mortar M2. Post-mortem analysis coupled with a statistical approach showed that the failure of M2S specimens was likely due to pores of the matrix [4]. 
Fig. 2 Mesostructure of the concretes with mortar M1 (top) and with mortar M2 (bottom)

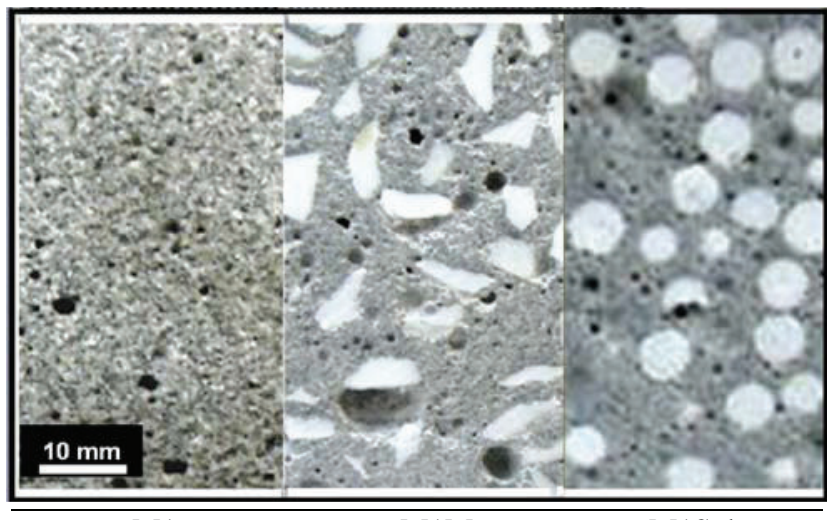

M1 M1M $\quad$ M1Sph

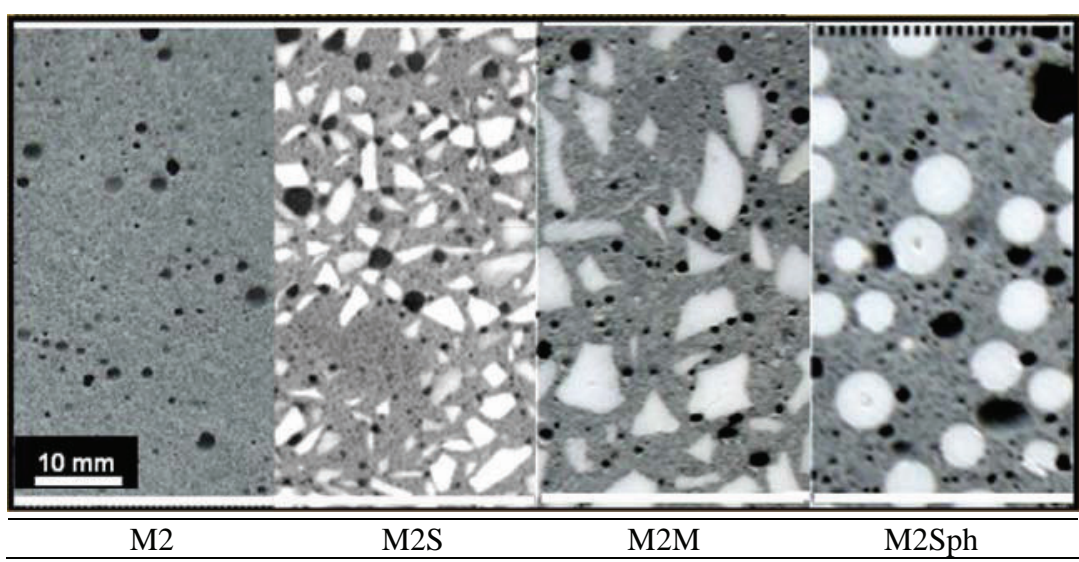

Table 1 Elementary properties of the seven materials

\begin{tabular}{|c|c|c|c|c|c|c|c|}
\hline Materials & M1 & M1M & M1Sph & M2 & $\mathrm{M} 2 \mathrm{~S}$ & $\mathrm{M} 2 \mathrm{M}$ & M2Sph \\
\hline Matrix & \multicolumn{3}{|c|}{ Without silica fume } & \multicolumn{4}{|c|}{ With silica fume } \\
\hline Shape of particles & & Angular & Spherical & & Angular & & Spherical \\
\hline Size of particles (mm) & & 36 & 4 & & 13 & 36 & 4 \\
\hline Vickers hardness HV $\left(\mathrm{kg} / \mathrm{mm}^{2}\right)$ & & 1000 & 1100 & & 1000 & & 1100 \\
\hline \multicolumn{8}{|l|}{ Mix proportions } \\
\hline Sand (quartz; kg/m³) & 1366 & 965.9 & & 1332 & 941.5 & & \\
\hline Silica fume $\left(\mathrm{kg} / \mathrm{m}^{3}\right)$ & & & & 55.5 & 39.2 & & \\
\hline Cement $\left(\mathrm{kg} / \mathrm{m}^{3}\right)$ & 569 & 402.5 & & 555 & 392.3 & & \\
\hline Water $\left(\mathrm{kg} / \mathrm{m}^{3}\right)$ & 260 & 183.5 & & 253 & 178.9 & & \\
\hline Admixture $\left(\mathrm{kg} / \mathrm{m}^{3}\right)$ & 4.7 & 3.4 & & 4.6 & 3.3 & & \\
\hline Alumina particles $\left(\mathrm{kg} / \mathrm{m}^{3}\right)$ & 0 & 1084.4 & & 0 & 1084.4 & & \\
\hline Water/(cement+silica fume) & 0.46 & & & 0.41 & & & \\
\hline Sand/(cement+silica fume $)$ & 2.4 & & & 2.2 & & & \\
\hline Silica fume/cement & & & & 0.1 & & & \\
\hline \multicolumn{8}{|l|}{ Densities } \\
\hline Measured density $\left(\rho_{\mathrm{C}} ; \mathrm{kg} / \mathrm{dm}^{3}\right)$ & 2.27 & 2.65 & 2.66 & 2.18 & 2.50 & 2.61 & 2.52 \\
\hline Mass fraction of particles $\left(f_{\mathrm{mP}}\right)$ & & 0.412 & 0.412 & & 0.412 & 0.412 & 0.412 \\
\hline Calculated density of the matrix $\left(\rho_{\mathrm{M}} ; \mathrm{kg} / \mathrm{dm}^{3}\right)$ & 2.27 & 2.24 & 2.26 & 2.18 & 2.06 & 2.19 & 2.09 \\
\hline \multicolumn{8}{|l|}{ Three point bending tests } \\
\hline Average failure stress (MPa) & 8.48 & 5.25 & 5.31 & 8.9 & 10.4 & 9.24 & 9.04 \\
\hline Number of specimens & 19 & 19 & 17 & 21 & 20 & 12 & 19 \\
\hline \multicolumn{8}{|l|}{ Simple compression tests } \\
\hline Average strength (MPa) & 59.3 & 38.3 & 29.1 & 66.8 & 76.0 & 70.8 & 42.4 \\
\hline Number of specimens & 4 & 4 & 2 & 4 & 3 & 2 & 2 \\
\hline
\end{tabular}


Table 1 also shows the strengths found under simple compression of the M1, M1M, and M1Sph mortars. The average failure stress of the M1M and M1Sph mixes is clearly below that of the M1 (half in the case of the M1Sph). Concerning the M2 matrix, angular particles were not perceptibly harmful to the compressive strength of the M2M and M2S grades, their average strength (Table 1) being even higher than that of the mortar M2 (without particles). As in bending, the angular particles did not lower the strength under uniaxial compression. The strength of the M2Sph specimens was clearly lower; however, a pullout of a few round particles during drilling may have weakened the M2Sph specimens.

\section{Determination of the Confined Compression Response}

Quasi-oedometric compression tests were run with each material according to the experimental method described ("Method of analysis of the quasi-oedometric compression test" sections). Figure 3 shows specimens after two tests of a cement-based mortar without particles and one containing ceramic particles. The four strain gauges are visible on the outer surface of the vessel. Their barrel strain is hardly perceptible. Part of the concrete without particles adhering to the top compression disk, but the one with particles remained intact. Also, the interface product Chrysor ${ }^{\circledR}$ was visible on the inner surface of the vessel.

The deviatoric and volumetric curves of the seven materials are shown in Figs. 4 (M1 matrix) and 5 (M2 matrix). The results with the two mortars without particles (M1 and M2) were discussed in a previous work [5] and are shown on the same plots to allow an easy comparison with the particulate composites. A notable increase in strength occurred with the hydrostatic pressure whatever the mortar grade. For example, under a hydrostatic pressure of $500 \mathrm{MPa}$ the strength was about $460 \mathrm{MPa}$ (M1) or $500 \mathrm{MPa}$ for the M1M and M1Sph grades to be compared with the strength under simple compression, 59.3, 38.3 and $29.1 \mathrm{MPa}$, respectively, for the M1, M1M and M1Sph grades. Similar results are visible with the M2 mortar. A great increase in strength versus pressure was found. The M2 strength reached $370 \mathrm{MPa}$ under $450 \mathrm{MPa}$ of hydrostatic pressure, the strengths of M2Sph, M2S and $\mathrm{M} 2 \mathrm{M}$ increased up to about 525, 540 and $620 \mathrm{MPa}$, respectively, under a hydrostatic pressure of $550 \mathrm{MPa}$.

\section{Deviatoric Behaviour}

Without a doubt, Figs. 4 and 5 reveal the influence of the addition of alumina particles in a cement-based matrix made with or without silica fume. Concerning the M1 matrix, oppositely to the bending and compression tests, addition of alumina particles had a noticeable positive effect on the strength of the M1M and M1Sph mortars under confinement. The beneficial effect of adding alumina particles on the deviatoric strength is even more spectacular with the matrix M2, regardless of the particle type. Moreover, particles influenced the shape of the curve of deviatoric stress versus pressure. For example, above $400 \mathrm{MPa}$ of hydrostatic pressure, the deviatoric stress of mortar without particles M2 showed a saturation of strength about $360 \mathrm{MPa}$. This tendency was not noted in any particulate mortars (M2S, M2Sph and M2M). This result should be confirmed under higher pressure of confinement but it appears to indicate that ceramic aggregates still acted as reinforcement for the highest pressure, up to $550 \mathrm{MPa}$, and for the highest deviatoric strain levels, about $12.5 \%$, $13.5 \%$ for the M2M and M2S materials, respectively, and even $15.5 \%$ for the M2Sph at the end of the tests.

A noteworthy point is that not all the particulate composites behaved in the same way. In fact, the slope of the curve (deviatoric stress vs. pressure) differs for three materials (M2Sph, M1M and M1Sph) approximately at the same point, a pressure of about $200 \mathrm{MPa}$ and a deviatoric

Fig. 3 View of two vessels after quasi oedometric compression tests

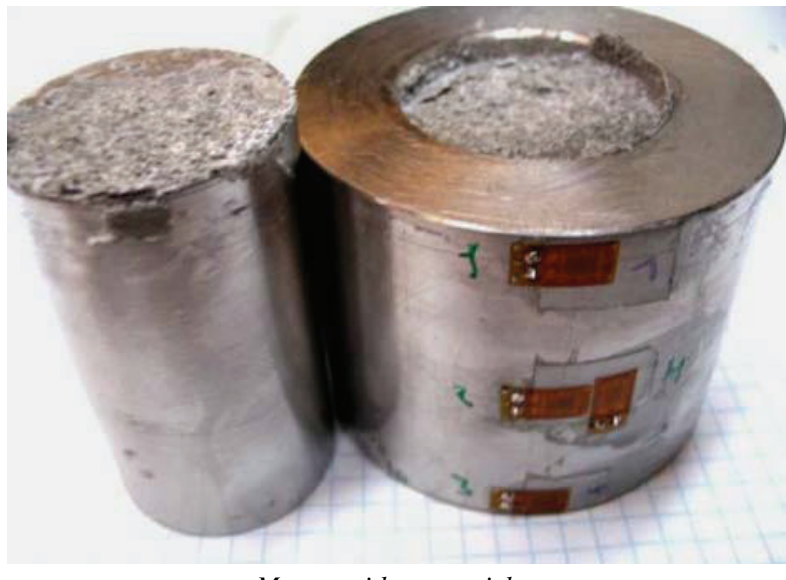

Mortar without particles

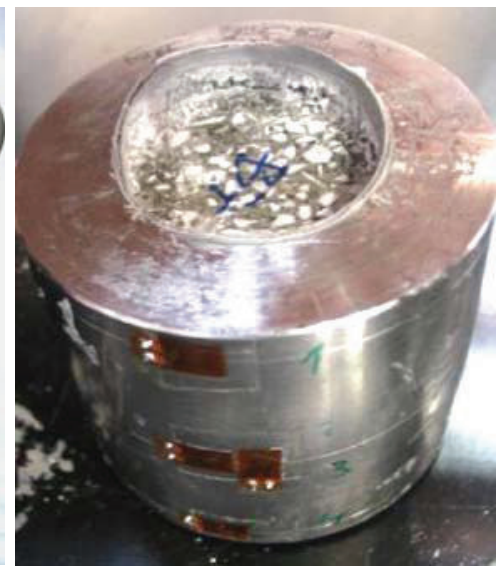

Mortar with angular particles 
Fig. 4 Deviatoric and volumet ric behaviour of mortars M1 without particles or with angular or spherical particles (M1M, M1Sph)

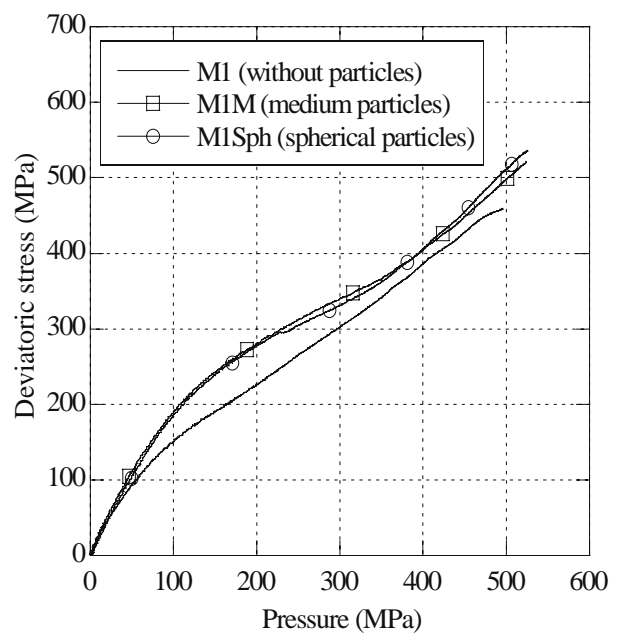

Deviatoric behaviour: evolution of the strength with hydrostatic pressure

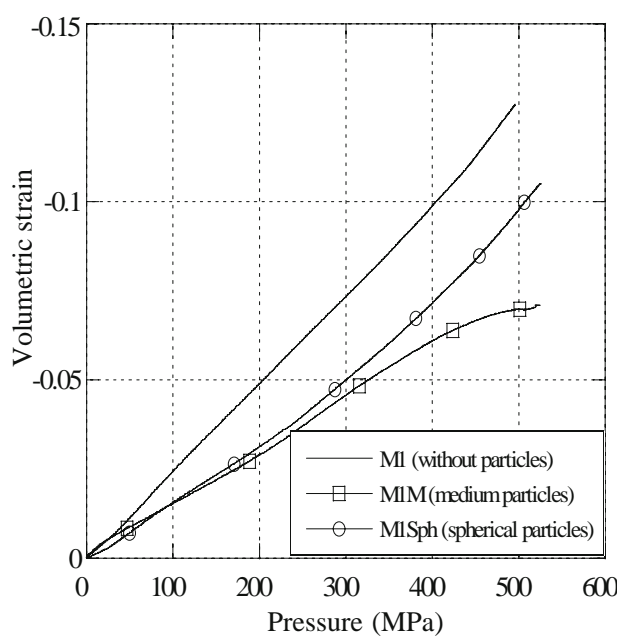

Volumetric behaviour: evolution of the volumetric strain with hydrostatic pressure stress about $300 \mathrm{MPa}$, while this change of slope was not observed in the $\mathrm{M} 2 \mathrm{~S}$ and $\mathrm{M} 2 \mathrm{M}$ grades. The highest strength under high confining pressure was found with the M2M grade. Angular particles led to higher strength than spherical particles, medium-size particles were preferable to small particles, and the addition of silica fume in the matrix was beneficial especially with angular particles.

\section{Volumetric Behaviour}

Again, it appears that the presence of particles had a favourable influence on the compaction curves of the cement-based materials. The volume reduction was less with the ceramic aggregates. For example, under a hydrostatic pressure of $400 \mathrm{MPa}$, the reduction of volume in $\mathrm{M} 1$ concretes appeared to be less than $30 \%$ when angular or spherical particles were used. Similarly, the volume of the M2 specimen was reduced by about $12 \%$ under a hydrostatic pressure of $400 \mathrm{MPa}$, while it fell below $10 \%$, $9.5 \%$, and $8 \%$, respectively, for the M2S, M2Sph, and the M2M materials under the same load. Thus, the presence of the particles can be highly beneficial, especially with mortars M2M, M2Sph, M1M and M1Sph.

Moreover, a strong correlation is observed between the porosity of the matrix and the compaction law of the material. First, in accordance with the measurements of densities (Table 1), the reduction of volume was higher with the M2 mortar than with M1, probably a consequence of the millimetre-length porosity in the M2 material [5]. The same results were found with mortars containing particles, in accordance with the different densities of their matrix.

Influence of Friction and Interface Product

Numerical simulations of the quasi-oedometric test of concrete specimens were made with Abaqus/Explicit for
Fig. 5 Deviatoric and volumet ric behaviour of mortars M2 without particles or with angular (small or medium size) particles (M2S, M2M) or with spherical particles (M2Sph)

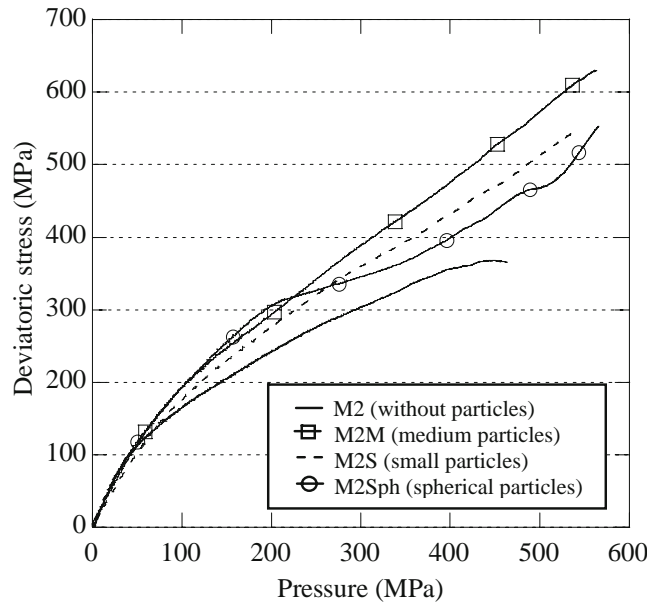

Deviatoric behaviour: evolution of the strength with hydrostatic pressure

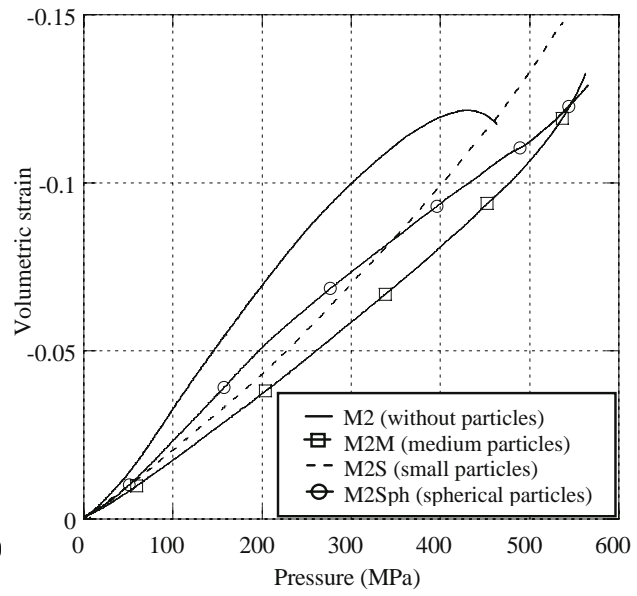

Volumetric behaviour: evolution of the volumetric strain with hydrostatic pressure 
Table 2 Parameters of M2S concrete in the Krieg, Swenson and Taylor model

\begin{tabular}{lll}
\hline Parameters & & \\
\hline $\begin{array}{l}\text { Density, elastic parameters } \\
\text { Volumetric parameters }\end{array}$ & $\rho, E, \nu$ & $2.386,46 \mathrm{GPa}, 0.2$ \\
$\begin{array}{c}\text { Compaction curve } \\
\text { (three points) }\end{array}$ & $\varepsilon_{v}^{(i)}, P_{(i)}^{(i)}$ & $0.0003,7.67 \mathrm{MPa}$ \\
& $\left.\varepsilon_{v}^{(i)}, P_{(i}^{(i)} 2\right)$ & $0.042,200 \mathrm{MPa}$ \\
& $\left.\varepsilon_{v}^{(i)}, P_{(i)}^{(i)} 3\right)$ & $0.15,580 \mathrm{MPa}$ \\
$\begin{array}{l}\text { Deviatoric parameters } \\
\text { Coefficient of elliptical } \\
\text { equation }\end{array}$ & $a_{012}$ & $625 \mathrm{MPa}^{2}, 270 \mathrm{MPa}, 0.505$ \\
\hline
\end{tabular}

analysis of experimental procedure. The Abaqus/Explicit FE code [39] was selected to benefit from a user subroutine 'Vumat' of the Krieg, Swenson, and Taylor model [40, 41]. This model describes the volumetric behaviour by a compaction law and it has been used previously in confined concrete $[5,34]$ for checking the influence of test conditions on the stress and strain field. This compaction law links the volumetric strain to the hydrostatic pressure by means of a piece-wise linear function defined by several points $\left(\varepsilon_{v}^{i}, p_{i}\right)$ (Table 2). The model is accompanied by a limitation of the equivalent stress $\sigma_{\text {eq }}$ (von Mises criterion) according to the hydrostatic pressure [ $p$; equation (8)].

$\sigma_{\mathrm{eq}}=\sigma_{y}(p)=\sqrt{ } a_{0}+a_{1} p+a_{2} p^{2}$

These various coefficients $\left(a_{0}, a_{1}, a_{2}, \varepsilon_{v}^{i}, p_{i}\right)$ were identified for the M2S material (Fig. 6, Table 2). The plastic strain tensor $\mathbf{e}^{p}$ is defined as

$\mathbf{e}^{p}=\mathbf{e}-\frac{\mathbf{s}}{2 G}$

where $\mathbf{e}$ is the deviatoric part of the total strain tensor, $\mathbf{s}$ the deviatoric stress tensor, and $G$ the shear modulus. The plastic strain increment $\mathrm{de}^{p}$ is given by

$\mathrm{de} \mathbf{e}^{p}=\mathrm{d} \lambda \frac{\partial \phi_{s}}{\partial \mathbf{s}}$

$\phi_{s}$ being a non-associative plastic potential given by $\phi_{s}=\sigma_{\text {eq }}$.
In numerical simulations, a uniform axial velocity is applied to the upper surface of the compression plug, at a rate low enough to ensure a load similar to a quasi-static load (the rate having no effect on the result of the numerical simulation). We used reduced integration axisymmetrical elements (CAX4R in ABAQUS notation). The numerical simulation allowed us to check the homogeneity of radial stress field at the specimen/vessel contact, thereby validating the hypothesis used in the analysis method [equation (3)].

Figure 7 presents the result of the processing of data of the seven numerical simulations of a quasi-oedometric compression test. The left side of Fig. 7 shows the deviatoric behaviour and the right-hand column the volumetric behaviour. The parameters used for the concrete are those given in Table 2. The processing of data is applied in the same way as experimental data would be processed. First, axial force and displacement of the plug are used to deduce the average axial stress and strain in the specimen. According to equation (3) the radial stress is computed from external hoop strain of the vessel and from the axial strain. Neither influence of friction nor that of the Chrysor ${ }^{\circledR}$ interface product is considered in the processing. This hypothesis is discussed below. The error committed in the evaluation of the strength and of the volumetric strain is reported in Table 3 for each numerical simulation.

The first numerical simulation of Fig. 7 corresponds to a quasi-oedometric compression test without any friction or play, either at the specimen/vessel interface or at the specimen/compression plate interface. The "measured" volumetric and deviatoric behaviour found by applying the method of processing of equations (3), (4), (5), (6), and (7) coincides very well with the behaviour indicated in the numerical simulation for the specimen (KST model). In the numerical simulations no. 2 and no. 3 , a friction coefficient was used at the vessel/specimen interface $(0.1$ and 0.2 , respectively). The error committed in the evaluation of the strength remained below $4 \%$ but that in the evaluation of the volumetric strain reached $11.3 \%$ and $18.6 \%$, respec-
Fig. 6 Identification of KST parameters for M2S material
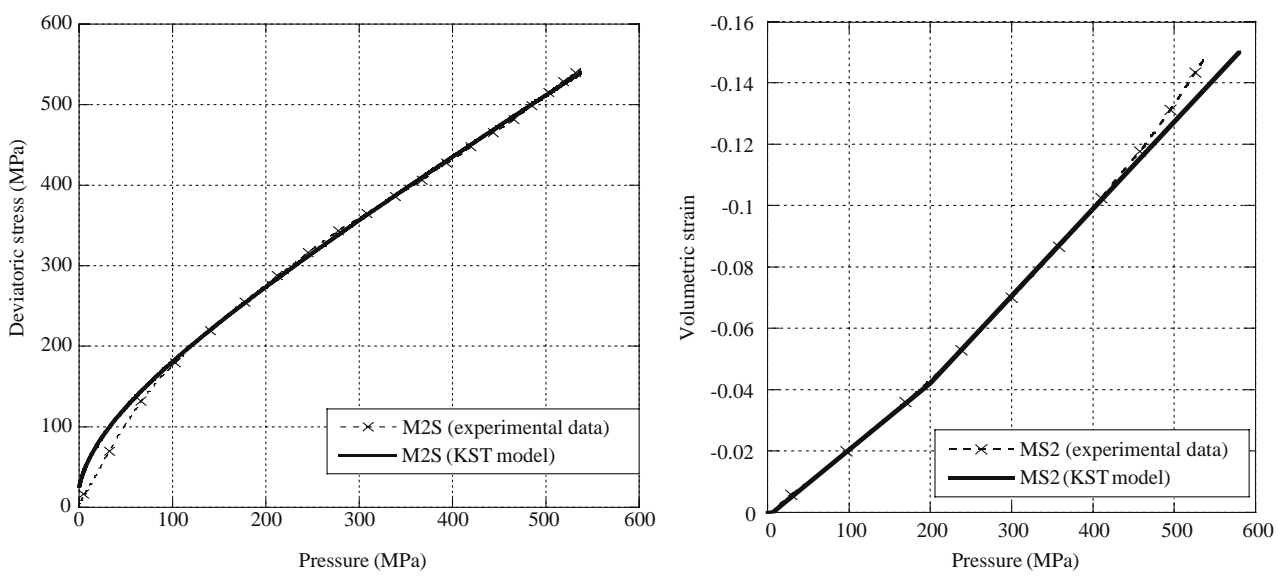
Fig. 7 Processing of data from numerical simulation of the quasi oedometric compression test. $\left(f_{\mathrm{p} / \mathrm{s}}:\right.$ friction $\mathrm{plug} / \mathrm{specimen}$, $f_{\mathrm{v} / \mathrm{s}}:$ friction vessel/specimen, with Chrysor: $0.3 \mathrm{~mm}$ filled with Chrysor ${ }^{\circledR}$ product)
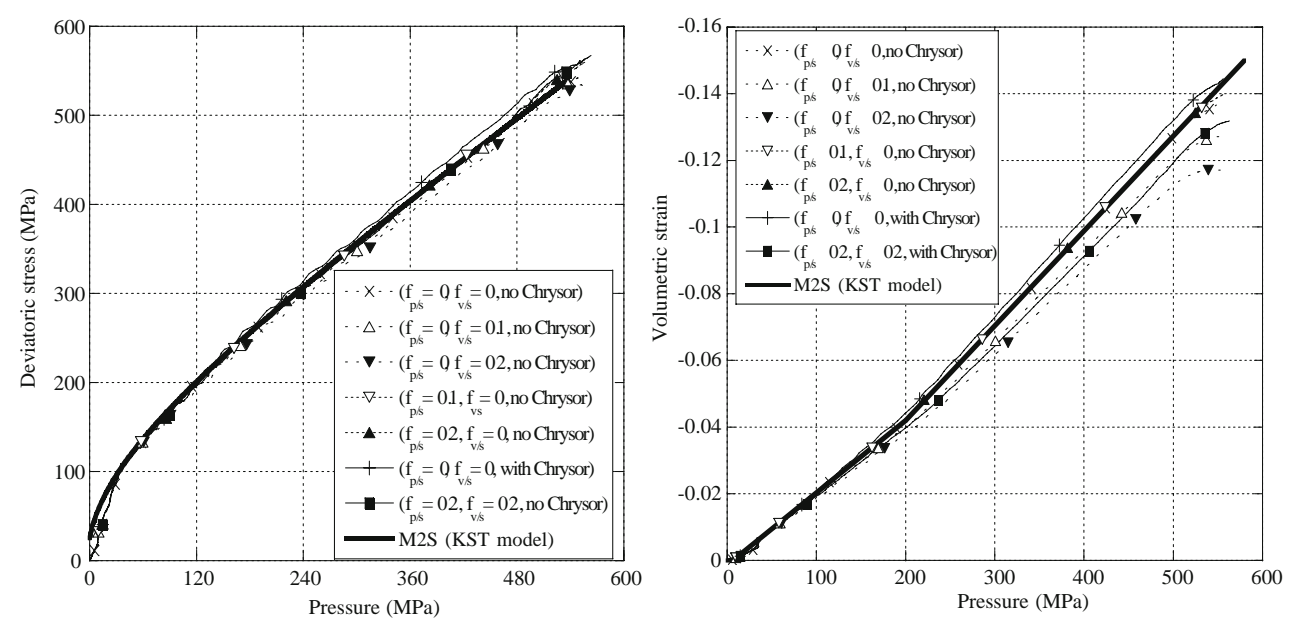

tively, for friction coefficients of 0.1 and 0.2 when the axial strain was equal to $-20 \%$. In the numerical simulations $n^{\circ} 4$ and $\mathrm{n}^{\circ} 5$ the contact at the vessel/specimen interface is assumed without friction but a friction coefficient is considered at $\mathrm{plug} / \mathrm{specimen}$ interface, $(0.1$ and 0.2 , respectively). Both deviatoric and volumetric behaviour was appreciably unaffected and the error remains as low as that in the numerical simulation without friction (case no. 1). In the numerical simulations no. 6 and no. 7 it was assumed that the Chrysor ${ }^{\circledR}$ resin was filling a gap of $3 /$ $10 \mathrm{~mm}$ between the specimen and the vessel. No friction was considered in the case no. 6 , whereas in the case no. 7 a friction coefficient of 0.2 was assumed at both interfaces (plug/specimen and Chrysor ${ }^{\circledR}$ product/vessel). An isotropic elastic behaviour was considered to model the Chrysor ${ }^{\circledR}$ resin (Young modulus: 2.2 GPa, Poisson ration: 0.28) [12]. These calculations confirm that the test-analysis method was not in itself the source of an error above $4 \%$ in the deviatoric behaviour and one of $12 \%$ in the volumetric behaviour whether the friction at the vessel/specimen interface remained below 0.1 .

In evaluating the friction at vessel/specimen interface, we found that the barrelling deformation of the vessel increased when friction acted on its internal surface. The

Table 3 Difference between the expected behaviour (imposed for the specimen in the computation, Table 2) and that deduced from the processing of data of numerical simulation for three axial strains

\begin{tabular}{|c|c|c|c|c|c|c|}
\hline $\begin{array}{l}\text { Numerical } \\
\text { simulation }\end{array}$ & $\begin{array}{l}\text { Friction plug/ } \\
\text { specimen }\left(f_{\mathrm{p} / \mathrm{s}}\right)\end{array}$ & $\begin{array}{l}\text { Friction vessel/ } \\
\text { specimen }\left(f_{\mathrm{v} / \mathrm{s}}\right)\end{array}$ & $\begin{array}{l}\text { Chrysor }^{\circledR} \\
\text { interface }\end{array}$ & $\begin{array}{l}\text { Nominal axial } \\
\text { strain }(\%)\end{array}$ & $\begin{array}{l}\text { Error deviatoric } \\
\text { stress }(\%)\end{array}$ & $\begin{array}{l}\text { Error volumetric } \\
\text { strain }(\%)\end{array}$ \\
\hline \multirow[t]{3}{*}{1} & \multirow[t]{3}{*}{0} & \multirow[t]{3}{*}{0} & \multirow[t]{3}{*}{ No } & 10 & 0.5 & 0.2 \\
\hline & & & & 15 & 0.7 & 0.2 \\
\hline & & & & 20 & 1.2 & 3.7 \\
\hline \multirow[t]{3}{*}{2} & \multirow[t]{3}{*}{0} & \multirow[t]{3}{*}{0.1} & \multirow[t]{3}{*}{ No } & 10 & 1.2 & 5.9 \\
\hline & & & & 15 & 0.3 & 6.3 \\
\hline & & & & 20 & 1.5 & 11.3 \\
\hline \multirow[t]{3}{*}{3} & \multirow[t]{3}{*}{0} & \multirow{3}{*}{0.2} & \multirow{3}{*}{ No } & 10 & 3.1 & 11.3 \\
\hline & & & & 15 & 1.9 & 12.9 \\
\hline & & & & 20 & 3.3 & 18.6 \\
\hline \multirow[t]{3}{*}{4} & \multirow[t]{3}{*}{0.1} & \multirow[t]{3}{*}{0} & \multirow[t]{3}{*}{ No } & 10 & 0.1 & 0.1 \\
\hline & & & & 15 & 1.6 & 0.4 \\
\hline & & & & 20 & 1.1 & 2.6 \\
\hline \multirow[t]{3}{*}{5} & \multirow[t]{3}{*}{0.2} & \multirow[t]{3}{*}{0} & \multirow[t]{3}{*}{ No } & 10 & 0.1 & 0.1 \\
\hline & & & & 15 & 1.7 & 0.4 \\
\hline & & & & 20 & 1.1 & 2.6 \\
\hline \multirow[t]{3}{*}{6} & \multirow[t]{3}{*}{0} & \multirow[t]{3}{*}{0} & \multirow[t]{3}{*}{ Yes } & 10 & 2.4 & 3.8 \\
\hline & & & & 15 & 3.4 & 3.7 \\
\hline & & & & 20 & 1.7 & 0.9 \\
\hline \multirow[t]{3}{*}{7} & \multirow[t]{3}{*}{0.2} & \multirow[t]{3}{*}{0.2} & \multirow[t]{3}{*}{ Yes } & 10 & 0.1 & 7.7 \\
\hline & & & & 15 & 2.4 & 6.0 \\
\hline & & & & 20 & 1.4 & 9.2 \\
\hline
\end{tabular}


barrelling deformation of the vessel may be characterised by the ratio of hoop strains measured on the external surface of the vessel $\left(\varepsilon_{\theta \theta}^{(z=18)} / \varepsilon_{\theta \theta}^{(z=0)}\right)$ or by the ratio of axial strain to hoop strain in the symmetry plane $\left(\varepsilon_{z z}^{(z=0)} / \varepsilon_{\theta \theta}^{(z=0)}\right)$. Both ratios may be deduced from experimental data. As shown by Fig. 8, the higher the friction the lower the ratio of hoop strains $\left(\varepsilon_{\theta \theta}^{(z=18)} / \varepsilon_{\theta \theta}^{(z=0)}\right)$ and the higher the ratio of axial to hoop strains $\left(\varepsilon_{z z}^{(z=0)} / \varepsilon_{\theta \theta}^{(z=0)}\right)$. Moreover, the friction at plug/specimen interface (noted $\mathrm{f}_{\mathrm{p} / \mathrm{s}}$, Fig. 8) as well as the Chrysor ${ }^{\circledR}$ interface product had a very little or no influence on both ratios $\left(\varepsilon_{\theta \theta}^{(z=18)} / \varepsilon_{\theta \theta}^{(z=0)}\right.$, $\left.\varepsilon_{z z}^{(z=0)} / \varepsilon_{\theta \theta}^{(z=0)}\right)$. On the contrary, friction at the vessel/ specimen interface (noted $f_{v / s}$, Fig. 8) clearly affected the same ratios. Thus, their evolution as a function of the axial strain may be used to evaluate the level of friction at vessel/ specimen interface. However, as the level of axial strain remains quite low during the test (less than $30 \%$ of the hoopstrain level) the second ratio $\left(\varepsilon_{z z}^{(z=0)} / \varepsilon_{\theta \theta}^{(z=0)}\right)$ gave probably a less reliable value for the friction coefficient $f_{\mathrm{v} / \mathrm{s}}$. To conclude, according to Fig. 8, this friction coefficient is lower than 0.1 .

\section{Post-mortem Study of Quasi-oedometric Compression Tests}

A post-mortem study was made of the seven quasioedometric compression tests (Figs. 9, 10 and 11). Firstly, the steel confinement vessel was cut and the specimens were coated on both sides of the vessel with a highly fluid coloured resin. Latter, the specimens were cut along the symmetry plane and given a light polish. The following general considerations are relevant:

- In all specimens, the fracture surface showed a high density of cracks, mostly perpendicular to the axis of compression.
- The thickness of the interface product Chrysor ${ }^{\circledR}$ appeared to be more or less constant, showing that the specimens did not make contact with the vessel during the operation.

- The three close-ups of Fig. 11 (M2Sph, M2M, and $\mathrm{M} 2 \mathrm{~S}$ ) show that the product was not perforated or crushed by the angular or spherical particles on the cylindrical surface, so that it withstood the lateral compression during the test.

- No opened pores were visible in the post-mortem studies (Figs. 9, 10, 11), whereas their presence was evident in the mesostructures of Fig. 2. Pores, at least those of millimetre size, were totally collapsed by the confined compression.

\section{Cracking Stage}

Cracks were due not only to the presence of particles, but appeared abundantly also in the M1 and M2 grades (closeups of Fig. 9). The high density of cracking within the specimens and above all the intense transgranular fracturing of angular alumina particles, especially in M2M and M2S, raises doubt as to whether that cracking actually occurred during loading or on unloading. Indeed, the compressive radial stress field applied by the elastic release of the vessel during unloading cannot exceed a level of $300 \mathrm{MPa}$. This compressive loading level is incapable of inducing such cracking of alumina particles [42].

Concerning M2M and M2Sph, the cracks seem to be concentrated mainly near the ends of the specimen. In fact, the most promising hypothesis is that some horizontal cracks really exist also in the central part of these specimens but they are very difficult to see. The friction between the specimen and the vessel probably played a confinement role during the unloading, thereby preventing the cracks from opening in the centre.
Fig. 8 Influence of friction (plug/specimen, vessel/specimen) on the strain gauge response (left hand side: ratio of external hoop strains, right hand side: ratio of axial strain to hoop strain)
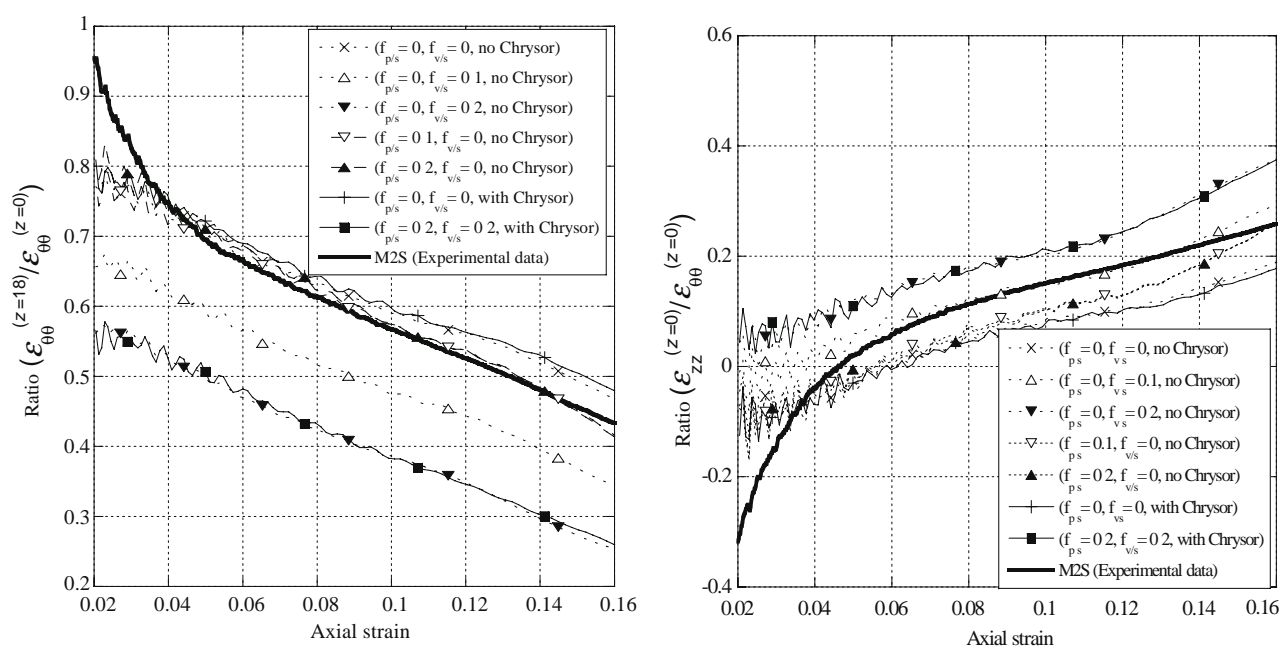


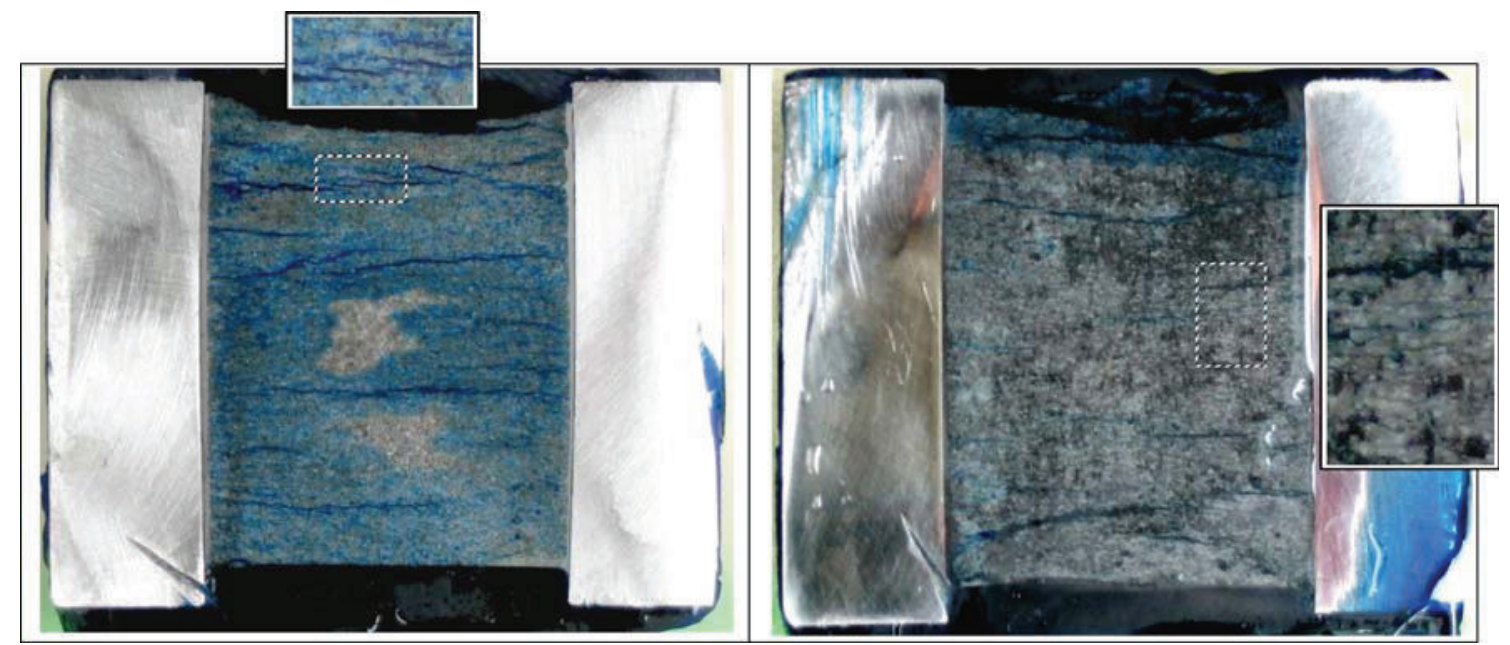

Fig. 9 Post mortem studies of quasi oedometric compression tests of mortars without particles (M1: left hand side and M2: right hand side)

In conclusion, the damage patterns are likely the result from the loading stage and the failure mechanism is one of mode II multiple cracking with rubbing lips for which the kinetics remain unclear. In fact, a similar damage mechanism was seen during impact tests with axial confinement and during edge-on impact tests with an ultra-highperformance concrete [43, 44].
Cement Composites Without Alumina Particles

Post-mortem studies of mortars without particles have shown an intense damage of numerous quasi-horizontal cracks (Fig. 9). The crack lengths in M1 ranged from a few millimetres to a few centimetres. As shown in the close-up of Fig. 9 (left), cracks seem to stop when they get too close

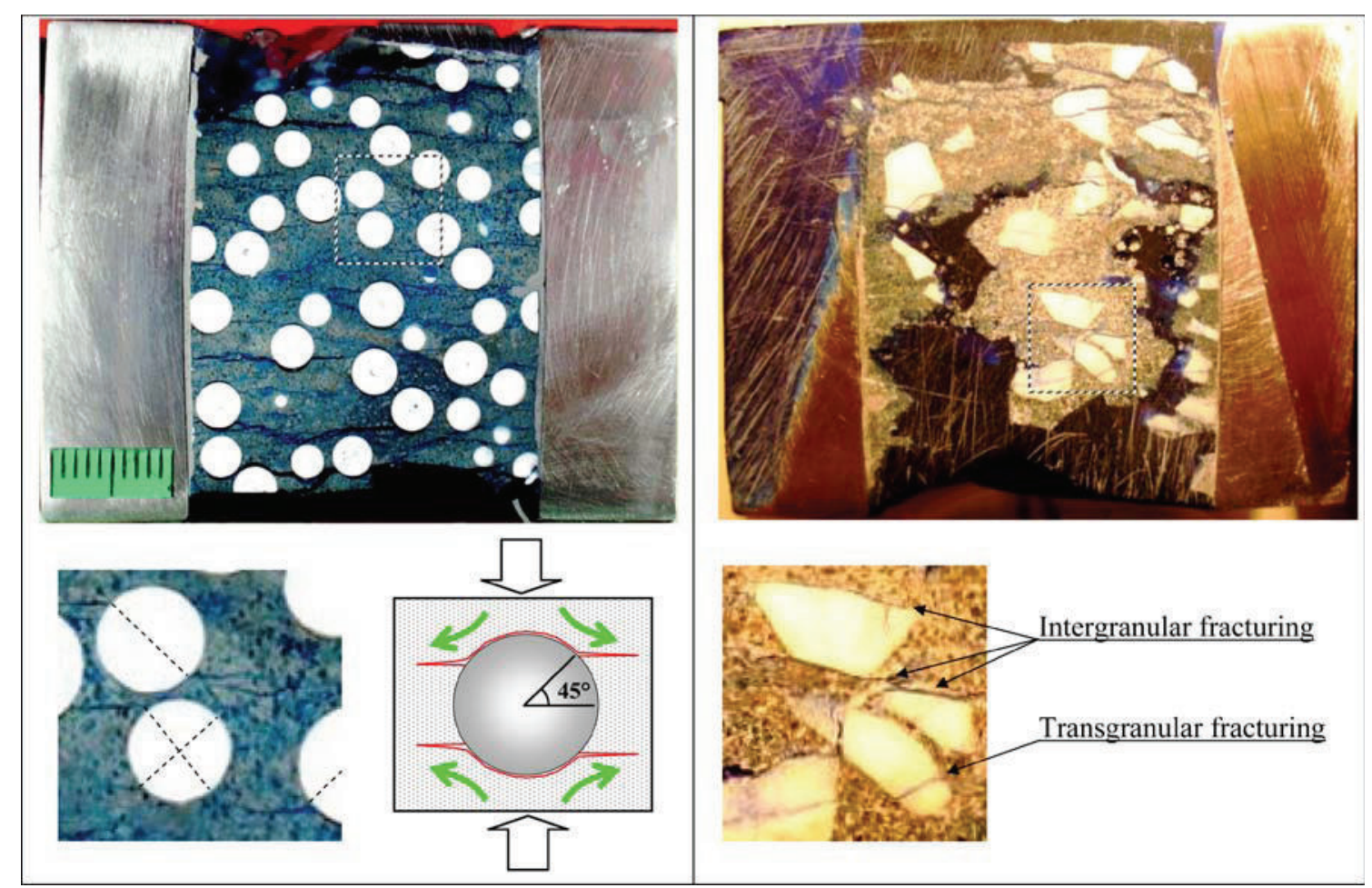

Fig. 10 Post mortem studies of quasi oedometric compression tests of mortar M1 with spherical or angular particles (M1Sph, M1M) 


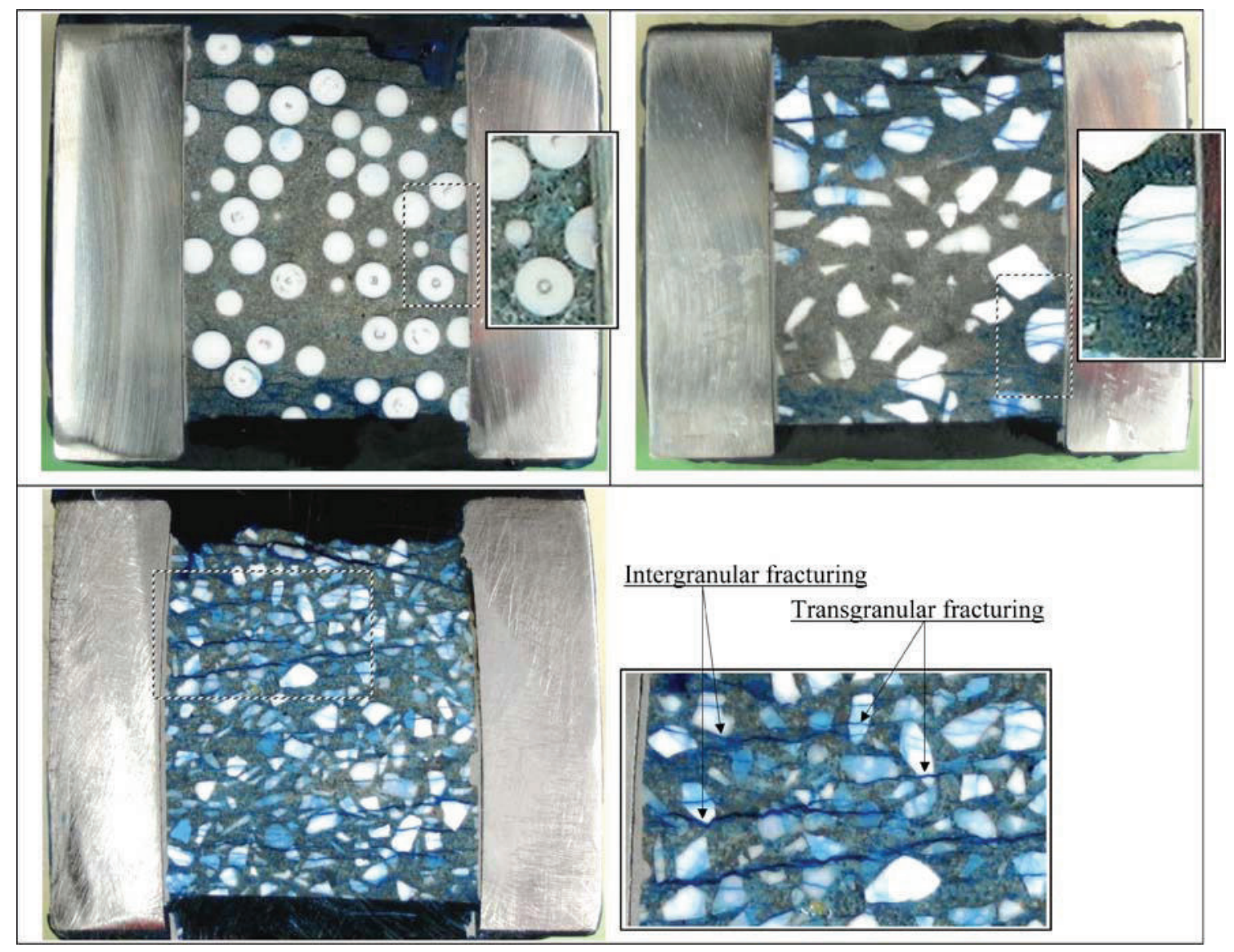

Fig. 11 Post mortem studies of quasi oedometric compression tests of mortar M2 with spherical particles (top left view), with angular particles of medium size (top right view) and with small sized angular particles (bottom view)

to each other. The close-up from M2 (right-hand side) reveals also a very high density of cracking with cracks as close as a few tenths of a millimetre. The difference of cracking densities between mortars prompts us to propose a hypothesis concerning the origin of cracking. The crushing of the millimetre porosity, especially in the M2 mortar, might trigger microcracks from pores. This would underscore the high damage level of the M2 mortar and also its weak deviatoric and volumetric behaviour (Fig. 5) during the quasi-oedometric compression test.

\section{Cement Composites Containing Alumina Particles}

Post-mortem studies of mortars containing medium-size particles show different failure patterns (Figs. 10 and 11). The angular particles and the spherical ones did not work in the same way during the tests: the angular alumina particles (M1M and M2M materials) appear to be sheared by the horizontal cracks that travel through the specimens. Concerning the M1Sph and M2Sph grades, only the matrix is cracked and the spherical alumina particles are intact. As a consequence of their smaller size and their rounder shape, spherical particles were twisted by the numerous horizontal cracks while angular particles were crossed over. The density of cracking of the M1Sph specimen was much greater than the ones visible within the M2Sph and the $\mathrm{M} 2 \mathrm{M}$ specimens. We might imagine that some spherical particles in the M1Sph grade initiated at least a part of the cracking in this specimen. This hypothesis is reinforced by the fact that the numerous short cracks were systematically connected at $45^{\circ}$ regarding the centre of the spherical particles (Fig. 10). Finally, the fact that the flow of matrix around the angular particles was hindered by their shape could explain the lower density of cracking (for M1M in comparison with $\mathrm{M} 1 \mathrm{Sph}$ ) and the transgranular fracturing observed with these particles (for M1M and M2M grades in comparison with M1Sph and M2Sph grades).

Thus, the deviatoric strength of the M2M mortars with angular particles was higher than that of the mortars with spherical particles M2Sph under a pressure of some $400 \mathrm{MPa}$ because the angular particles offered a longer strength to shear. On the other hand, it may highlight why the deviatoric and volumetric behaviour of the cement composites containing medium-size particles were similar at the end of the loading, the efficiency of angular particles being greatly diminished when fractured. In fact, the 
singular evolution of deviatoric stress versus pressure noticed previously for the M1M, M1Sph, M2Sph might be the consequence of a particle/matrix decohesion fracturing the matrix, as illustrated by the two close-ups in Fig. 10. This decohesion may be explained by the poor interfacial bond strength for the M1M mortar or the unfavourable shape of spherical particles (M2Sph mortar) or both effects (M1Sph mortar). At the same time, this hypothesis could explain also why this effect was not observed between 200 and $400 \mathrm{MPa}$ of hydrostatic pressure in the mortars without particles (M1 and M2).

Concerning the M2S material (Fig. 11), we can see an intense cracking of the specimen, with many short and long cracks (length in the range of few $\mathrm{mm}$ to few $\mathrm{cm}$ ). This high density of cracking highlights quite well the low deviatoric strength observed for this material. The fracturing is mainly transgranular even if particles/matrix decohesion is visible. Two main hypotheses may be proposed to explain this damage pattern: particle breakage due to a bending state of particles or cracks initiated from a crushing of pores. Given that many particles are fractured on the edge, some particles are multi-cracked and some round particles are also fractured, the first hypothesis may be thought to be less probable. Moreover, the second hypothesis, an origin of cracking from crushed pores, is consistent with the high porosity level of this material, Table 1.

\section{Conclusion}

Quasi-oedometric compression tests were made with seven concrete grades containing or lacking angular or spherical alumina particles. The tests showed a highly beneficial effect of the presence of particles with respect to both the deviatoric strength and the compaction law. This has added interest because the standard tests (three-point bending and simple compression) did not show equivalent results (especially for the matrix without silica fume), proof that the strength of geomaterials not under confinement is not indicative of the behaviour of the same materials under confined loading. It was also found that the deviatoric strength is more favoured by angular particles than by spherical ones and by the addition of silica fume in the cement paste. In addition, a strong correlation was noted between the millimetre porosity and the compaction of the concretes under such levels of pressure.

Furthermore, an application of post-mortem analysis to confined tests enables us to determine the influence of mesostructure on mechanical properties under high pressures. The post-mortem study shows a damage field made of numerous cracks nearly perpendicular to the axial direction. However, noticeable differences were evident, depending on the mesostructure of the material considered.
In particular, particle/matrix decohesion appeared to be favoured in mortars lacking silica fume and in mortars containing spherical particles, whereas angular particles (of small and medium sizes) were abundantly fractured. Moreover, a strong correlation was found between the amount of porosity of millimetre-size and the amount of cracking in mortars without particles and in those containing angular particles. These findings shed light on the differences of confined strengths and volumetric behaviour observed between the different grades of concretes and on the role played by their mesostructure in controlling their mechanical behaviour under such loading.

Acknowledgements The authors are indebted to the Spanish Comisión Interministerial de Ciencia y Tecnología (Project MAT2002 03339), to the Comunidad Autónoma de Madrid and the University Carlos III of Madrid (CCG06 UC3M/DPI 0796 and CCG07 UC3M/DPI 3395) for the financial support of this work. To the Délégation Générale pour l'Armement (DGA/France) for the mobility grant provided to Dr. Forquin.

\section{References}

1. Malier Y (1992) Les bétons à hautes performances. In: Presses de l'Ecole Nationale des Ponts et Chaussées, France.

2. Cheyrezy M, Maret V, Frouin L (1995) Microstructural analysis of RPC. Cem Concr Res 257:1491 1500. doi:10.1016/0008 8846 (95)00143 Z.

3. Darrigade A, Buzaud E (1999) High performance concrete: a numerical and experimental study. In: Proceedings of the ninth international symposium on the interactions of the effects of munitions with structures. Berlin.

4. Arias A, Forquin P, Zaera R, Navarro C (2008) Relationship between bending and compressive behaviour of particle reinforced cement composites. Composites Part B. doi:10.1016/j. compositesb.2008.03.002.

5. Forquin P, Arias A, Zaera R (2008) Role of porosity in controlling the mechanical and impact behaviours of cement based materials. Int J Impact Eng 353:133 146. doi:10.1016/j.ijimpeng.2007.01.002.

6. Clifton RJ (2000) Response of materials under dynamic loading. Int J Solids Struct 37:105 113. doi:10.1016/S0020 7683(99) 000827.

7. Field JE, Walley SM, Proud WG, Goldrein HT, Siviour CR (2004) Review of experimental techniques for high rate deformation and shock studies. Int J Impact Eng 30:725 775. doi:10.1016/j. ijimpeng.2004.03.005.

8. Zhang MH, Shim VP, Lu G, Chew CW (2005) Resistance of high strength concrete to projectile impact. Int J Impact Eng 31:825 841. doi:10.1016/j.ijimpeng.2004.04.009.

9. Barr P (1990) Guidelines for the design and assessment of concrete structures subjected to impact. Atomic Energy Authority, London.

10. Hanchak SJ, Forrestal MJ, Young ER, Ehrgott JQ (1992) Perforation of concrete slabs with 48 and $140 \mathrm{MPa}$ unconfined compressive strength. Int J Impact Eng 121:1 7. doi:10.1016/ $0734743 \mathrm{X}(92) 90282 \mathrm{X}$.

11. Burlion N, Gatuingt F, Pijaudier Cabot G, Daudeville L (2000) Compaction and tensile damage in concrete: constitutive model ling and application to dynamics. Comput Methods Appl Mech Eng 183:291 308. doi:10.1016/S0045 7825(99)002236.

12. Forquin P, Arias A, Zaera R (2007) An experimental method of measuring the confined compression strength of geomaterials. Int 
J Solids Struct 4413:4291 4317. doi:10.1016/j.ijsolstr.2006. 11.022 .

13. Yankelevsky DZ, Dancygier AN (2001) Uniaxial compressive strength effect on high velocity penetration into thick NSC and HSC targets. Symposium ISIEMS 2001.

14. Xu Y, Keer LM, Luk VK (1997) Elastic cracked model for penetration into unreinforced concrete targets with ogival nose projectiles. Int J Solids Struct 3412:1479 1491. doi:10.1016/ S0020 7683(96)000996.

15. Bridgman PW (1952) Studies in large plastic flow and fracture. McGraw Hill, New York.

16. Paterson MS (1967) Effect of pressure on stress strain properties of materials. Geophys J R Astron Soc 14:13 17.

17. Heard HA, Cline CF (1980) Mechanical behaviour of Polycrys talline $\mathrm{BeO}, \mathrm{Al}_{2} \mathrm{O}_{3}$ and $\mathrm{AlN}$ at high pressure. J Math Sci 15:1889 1897.

18. Horii H, Nemat Nasser S (1986) Brittle failure in compression: splitting, faulting and brittle ductile transition. Philos Trans R Soc Lond 319:337 374. doi:10.1098/rsta.1986.0101.

19. Chen W, Ravichandran G (2000) Failure mode transition in ceramics under dynamic multiaxial compression. Int $\mathrm{J}$ Fract 101:141 159. doi:10.1023/A:1007672422700.

20. Ramsey JM, Chester FM (2004) Hybrid fracture and the transition from extension fracture to shear fracture. Nature 4286978:63 66 . doi:10.1038/nature02333.

21. Rittel D, Brill A (2008) Dynamic flow and failure fo confined polymethylmethacrylate. J Mech Phys Solids 56:1401 1416. doi:10.1016/j.jmps.2007.09.003.

22. Zimmerman RG (1972) Major factors affecting the multiaxial compressive strength of plain concrete. The deformations and the rupture of solids subjected to multiaxial stresses. In: Proc. of RILEM Int Symposium, Cannes, RILEM, Paris, 1:257 272.

23. Palaniswamy R, Shah SP (1974) Fracture and stress strain relationship of concrete under triaxial compression. J Struct Div ST5:901 916

24. Xie J, Elwi AE, MacGregor JG (1995) Mechanical properties of three high strength concretes containing silica fume. ACI Mater J 922:135 145

25. Bažant ZP, Xiang Y, Adley MD, Prat PC, Akers SA (1996) Microplane model for concrete. II: Data delocalization and verification. J Eng Mech 1223:255 262. doi:10.1061/(ASCE) 0733 9399(1996)122:3(255).

26. Buzaud E (1998) Performances mécaniques et balistiques du microbéton MB50. In: DGA/Centre d'Etudes de Gramat Report. France.

27. Wallace G, Olden OJ (1965) Foundation testing techniques for arch dams and underground developments. In: ASTM STP 402.

28. Ma Z, Ravi Chandar K (2000) Confined Compression: a stable homogeneous deformation for constitutive characterization. Exp Mech 40:38 45. doi:10.1007/BF02327546.
29. Rittel D, Hanina E, Ravichandran G (2007) A note on the direct determination of the confining pressure of cylindrical specimens. Exp Mech. doi:10.1007/s11340 00790708.

30. Huang C, Subhans G (2003) Influence of lateral confinement on dynamic damage evolution during uniaxial compressive response of brittle solids. J Mech Phys Solids 51:1089 1105. doi:10.1016/ S0022 5096(03)00002 4.

31. Bardia P, Narasimhan R (2006) Characterization of pressure sensitive yielding in polymers. Strain 42:187 196. doi:10.1111/ j.1475 1305.2006.00272.x.

32. Gatuingt F (1999) Prévision de la rupture des ouvrages en béton sollicités en dynamique rapide. In: Ph.D. Thesis, Ecole Normale Supérieure de Cachan, France.

33. Burlion N, Pijaudier Cabot G, Dahan N (2001) Experimental analysis of compaction of concrete and mortar. Int J Numer Anal Methods Geomech 25:1467 1486. doi:10.1002/nag.178.

34. Forquin P, Gary G, Gatuingt F (2008) A testing technique for concrete under confinement at high rates of strain. Int J Impact Eng 356:425 446. doi:10.1016/j.ijimpeng.2007.04.007.

35. Holmquist TJ, Johnson GR, Cook WH (1993) A computational constitutive model for concrete subjected to large strains, high strain rates, and high pressures. In: Proceedings of the fourteenth international symposium on the ballistics. Quebec.

36. Jovicic J, Zavaliangos A, Ko F (2000) Modeling of the ballistic behavior of gradient design composite armors. Compos Part A 31:773 784. doi:10.1016/S1359 835X(00)00028 2.

37. Arias A, Zaera R, López Puente J, Navarro C (2003) Numerical modeling of the impact behavior of new particulate loaded composite materials. Compos Struct 611:151 159. doi:10.1016/ S0263 8223(03)00038 2.

38. Hibbitt HD, Karlsson BI, Sorensen P (2003) Abaqus user's manual, ABAQUS/STANDARD.

39. Hibbitt HD, Karlsson BI, Sorensen P (2003) Abaqus user's manual, ABAQUS/EXPLICIT.

40. Krieg RD (1978) A simple constitutive description for soils and crushable foams. In: SC DR 7260883, Sandia National Laboratory. Report, USA.

41. Swenson DV, Taylor LM (1983) A finite element model for the analysis of tailored pulse stimulation of boreholes. Int J Numer Anal Methods Geomech 7:469 484. doi:10.1002/nag.1610070408.

42. Lankford J (1981) Temperature strain rate dependence of com pressive strength and damage mechanisms in aluminium oxide. $\mathrm{J}$ Mater Sci 16:1567 1578. doi:10.1007/BF02396874.

43. Forquin P (2003) Endommagement et fissuration de matériaux fragiles sous impact balistique, rôle de la microstructure. $\mathrm{In}$ : $\mathrm{PhD}$ Thesis, Ecole Normale Supérieure de Cachan, France.

44. Forquin P, Hild F (2007) Dynamic fragmentation of an ultra high strength concrete during edge on impact tests. J Eng Mech 1344:302 315. doi:10.1061/(ASCE)0733 9399(2008)134: $4(302)$. 